\title{
Angular Size-Redshift: Experiment and Calculation
}

\author{
V.R. Amirkhanyan ${ }^{1}$ \\ 1 Sternberg Astronomical Institute, Lomonosov Moscow State University, Moscow, 119992 Russia
}

\begin{abstract}
In this paper the next attempt is made to clarify the nature of the Euclidean behavior of the boundary in the angular size-redshift cosmological test. It is shown experimentally that this can be explained by the selection determined by anisotropic morphology and anisotropic radiation of extended radio sources. A catalogue of extended radio sources with minimal flux densities of about $0.01 \mathrm{Jy}$ at $1.4 \mathrm{GHz}$ was compiled for conducting the test. Without the assumption of their size evolution, the agreement between the experiment and calculation was obtained both in the $\Lambda C D M$ model $\left(\Omega_{m}=0.27, \Omega_{v}=0.73\right.$. $)$ and the Friedman model $(\Omega=0.1)$.

DOI: $10.1134 /$ S1990341314040026
\end{abstract}

\section{INTRODUCTION}

Apparently, Hoyle [1] was the first to note that in the Friedman space with $q>0$, the angular size $\Theta$ of an object with the extent of $\mathrm{D}$ at a redshift equal to about 1 attains a minimum. Thereby, the possibility to estimate the space geometry emerges. The first to conduct this test was Legg [2], who showed the relation between the 3CR catalog extended radio source angular sizes and the redshift. It turned out that the upper limit of the diagram, which corresponds to the longest extent of the radio sources of $400 \mathrm{kpc}$, shows the best agreement with the Euclidean geometry, not with the Friedman one. Realizing that this is absolutely impossible, Legg assumed that the linear sizes of the radio sources decrease with increasing redshift. In the Einstein-de Sitter model $(\mathrm{q}=0.5)$, he obtained the best agreement with the observations assuming the size evolution $D \sim(1+z)^{-1.5}$. In the same work Legg wrote prophetically that we observe not the true size of the radio source but its projection on the celestial sphere. Thus he asserted that the structure of the radio source is anisotropic and randomly oriented. Miley [3] and Hooley et al. [4], who used a wider sample of extended radio sources, obtained the same result. The most representative sample of 540 radio sources was used by Nilsson et al. [5] for the plotting the $\Theta-z$ diagram. It is mostly composed of the radio sources of the $3 \mathrm{CR}$ and $4 \mathrm{C}$ surveys. This work confirmed the predecessors' results: the boundary of the $\Theta$-z diagram is proportional to $1 /$ z. In 1982 Orr and Brown [6] assumed that the radio sources radiation pattern is not spherical: its maxima coincide with the direction of the jet. The authors constructed the diagram as axially symmetric to the radio source major axis which allowed them to make it a function of one argument: the orientation angle of the symmetry axis relative to the observer. This fruitful idea allowed them to abandon two types of radio sources (compact and extended) and consider the statistics of randomly oriented extended objects. At the conference in 1987 Amirkhanyan [7] showed that the non-spherical morphology, and the non-spherical radio source radiation connected with it, is inevitably accompanied by a simple selection effect, which imposes an upper limit on the apparent angular size of distant radio sources.

\section{SELECTION}

If the radiation and the structure of the object are not spherical, then the observed flux density $\mathrm{S}$ and the angular size $\Theta$ are defined not only by the luminosity Lv, the physical dimension $D_{0}$, and remoteness of an object $l_{v}$ but also by its orientation relative to the observer. The telescope can "see" the object if its flux density $\mathrm{S}$ exceeds the detection threshold $S_{t}$ :

$$
S=\frac{L_{v} \varphi(\phi)}{l_{v}^{2}}>S_{t}
$$

here $\varphi(\phi)$ describes the form of the radiation pattern. When $\phi=0$, the radiation toward the observer is maximal, and when $\phi=\pi / 2$, it is minimal. It follows from the detection condition (1) that such a

radio source will be seen whatever the orientation $(\phi=0 \div \pi / 2)$, up to the distance

$$
l_{t}=\sqrt{\frac{L_{v} \varphi(\pi / 2)}{S_{t}}}
$$


The selection appears with the distance increase, because the orientation angle of the radio source at which it could be observed decreases. Consequently, its projection on the celestial sphere decreases $\left(D=D_{0} \sin \phi\right)$. The upper boundary of the $\Theta$ - z diagram, which is usually calculated on the tacit assumption of spherically symmetric radio source radiation, at the distances greater than $l_{t}$ gets one more restriction in the form of the multiplier $\sin \phi_{\max }$. Knowing the form of the radio source radiation pattern [7, 8], one can easily obtain the dependence of the maximum angular size on redshift in the assumed space model and with a defined detection threshold. Such calculations were carried out in [7] for the 3CR catalogue, where the detection threshold is equal to $10 \mathrm{Jy}$ at the frequency of $\nu=178 \mathrm{MHz}$. As a result, the upper limit of the $\Theta-\mathrm{z}$ diagram close to $1 / \mathrm{z}$ was obtained in the Einstein.de Sitter model. In order to explain the selection mechanism, let us show the dependence of the orientation angle boundary on the redshift (see Fig. 1), plotting the radio source radiation pattern from [8]

$$
\varphi(\phi)=\frac{L}{L_{0}}=a(1+z)^{\alpha}+(1-a) \cos ^{2 n} \phi .
$$

Here $L_{0}$ is the luminosity toward the radiation peak; $a$ is the ratio of the luminosity of the isotropic component of the radiation to $L_{0}$ in the comoving coordinate system; $\alpha$ is the spectral index of the isotropic component; $\phi$ is the angle between the orientation of the radiation maximum and the observer's line of sight; $\mathrm{n}$ specifies the width of the main lobe of the radiation pattern. The calculations were done for the Friedman model with $\mathrm{q}=0.05$. The spectral index is $\alpha=-0.85\left(S \sim \nu^{\alpha}\right)$. We applied the parameters of the radiation pattern derived in this paper: $\mathrm{a}=0.005, \mathrm{n}=15$. The black line corresponds to the minimum flux density of $2 \mathrm{Jy}$ of the catalogue, the blue line is for 0.05 Jy. In Fig. 1 there is a flat region corresponding to arbitrary radio source orientations from 0 . to 90 .. The length of this region naturally increases with the decrease of the detection threshold. The extension of the redshift beyond the flat region is accompanied by the decrease of the maximum allowed orientation angle $\phi_{\max }$ and, as a consequence, by the radio source selection by this angle: the observer detects radio sources with the orientation less than $\phi_{\max }$, and this results in the limitation of the observed angular size. In view of the above, the routine question arises - what actually determines the apparent boundary of the $\Theta-z$ diagram: the cosmological evolution of the radio source sizes or the selection by the orientation angle?

\section{CATALOG}

To solve this dilemma, it is enough to reduce the detection threshold $S_{t}$, and thereby to defer the hypothetical selection to a higher $z$. If the radio sources stay under the boundary $1 / z$, it definitely means evolution. Hence the simple and natural conclusion follows: an extensive catalog of weak extended radio sources is needed. In order to defer the selection to $z \approx 1, S_{t}$ should be reduced by more than ten times. As there was no such catalog, it had to be made. It was compiled using the following data.

1. The catalog of double radio sources from Nilsson et al. [5]. Several extended 3CR radio sources were added to it. Note that in [5] the boundary is calculated for the radio sources' maximum size of $4 \mathrm{Mpc}$ assuming that the Hubble constant is $H_{0}=50 \mathrm{~km} / \mathrm{s} / \mathrm{Mpc}$. For the modern $H_{0}=71 \mathrm{~km} / \mathrm{s} / \mathrm{Mpc} /$ it corresponds to $2.8 \mathrm{Mpc}$. Further this value is used to calculate the $\Theta$ - z diagram boundaries.

2. Radio sources with known z from Machalski et al. [9]. An extended radio source from Machalski et al. [10] was added to this list.

3. Double QSOs from Buchalter et al. [11].

4. Extended radio galaxies at declinations greater than 60. from Lara et al. [12, 13].

5. Double SUMSS radio sources from Saripalli et al. [14].

6. Double QSOs from Amirkhanyan [15].

Moreover, by means of the modified programs from [15], the NVSS catalog [16] was analyzed, more than two thousand candidates for extended radio sources were formed and identified with the NED and SDSS optical objects. The programs are set up for searching the objects grouped by the word "double" even if their morphology is more complex. These programs cannot always cope with their task successfully if the object extent exceeds $200^{\prime \prime}$, as the probability of the projection of false radio and optical components onto the radio source image increases. Aiming to minimize the number of possible errors, the author looked through NVSS and FIRST [17] maps of the radio sources in which optical components with measured redshifts were found. If their morphology does not meet our concept of a classical "double" structure, they are excluded from our catalog. The redshifts of nine formed radio sources have been measured at the 6 -m telescope 


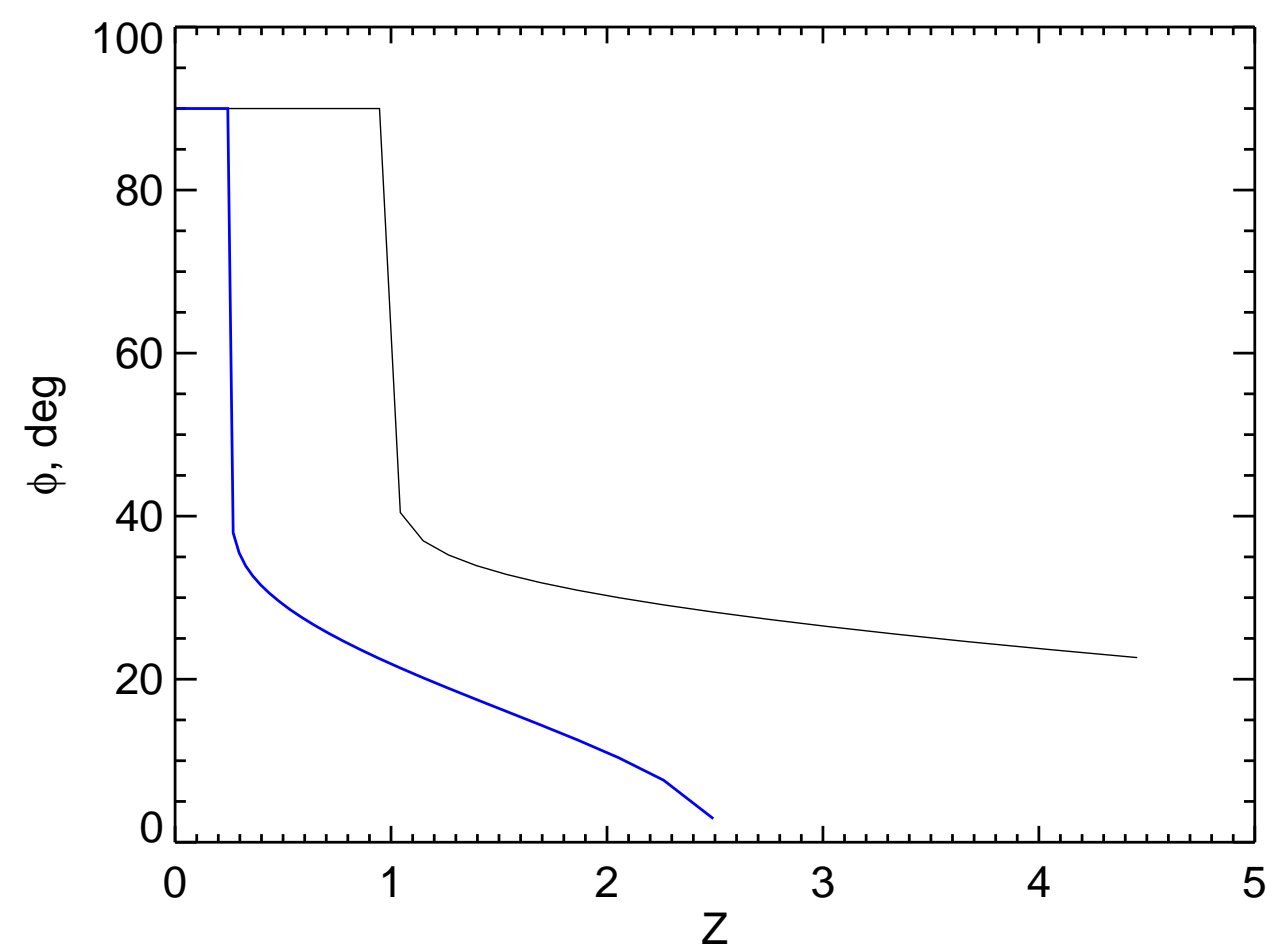

Figure 1. The dependence of the orientation angle's boundary from redshift for the two detection thresholds. Blue line- $S_{t}=2 \mathrm{Jy}$, black line $-S_{t}=0.05 \mathrm{Jy}$

of the Special Astrophysical Observatory (the paper is being prepared). Sixty-seven objects from this list crossed the conditional limit of $1 \mathrm{Mpc}$ (in the $\Lambda C D M$ space model currently accepted by the astronomical community) and noticeably expanded the giants list. As a result, 599 objects were added to the above mentioned lists. The total number of radio sources in the joint catalog is 1953: radio galaxies-913, quasars1040. The minimum flux densities are about $0.01 \mathrm{Jy}$ at the frequency of $1.4 \mathrm{GHz}$. If the radio source occurs in several of the above mentioned catalogs, the first publication is given the priority. The parameters (mainly redshift and angular size) of some of them are corrected according to the modern data. Some examples. The angular size of the 3C270 radio source in the Nilsson et al. [5] catalog is 498", and on the NVSS map its size is no less than $3000^{\prime \prime}$. The $3 \mathrm{C} 449$ object has the size of no less than $3600^{\prime \prime}$ on the NVSS map, although its catalog size is $300^{\prime \prime}$. The $3 \mathrm{C} 263.1$ redshift is 0.824 [18], not 0.366 .

\section{OBSERVATIONS AND CALCULATIONS}

Let us take the $\Theta-z$ diagram from [5] (Fig. 2) and

calculate its upper limit allowing for selection. For that we take the average model of the radio source radiation pattern from [8]. The integral of the diagram (3) over the full sphere differs from the standard $4 \pi$ [19]:

$$
h=4 \pi a(1+Z)^{\alpha}+2 \pi(1-a) \frac{(2 n-1) ! !}{(2 n) ! !}
$$

Hence, the full radio source luminosity

$$
L_{s}=h L_{0}
$$

The author experimented with several models of the radiation pattern, but this did not add any fundamental changes to the final result. The following parameter values have been used while calculating: the Hubble 


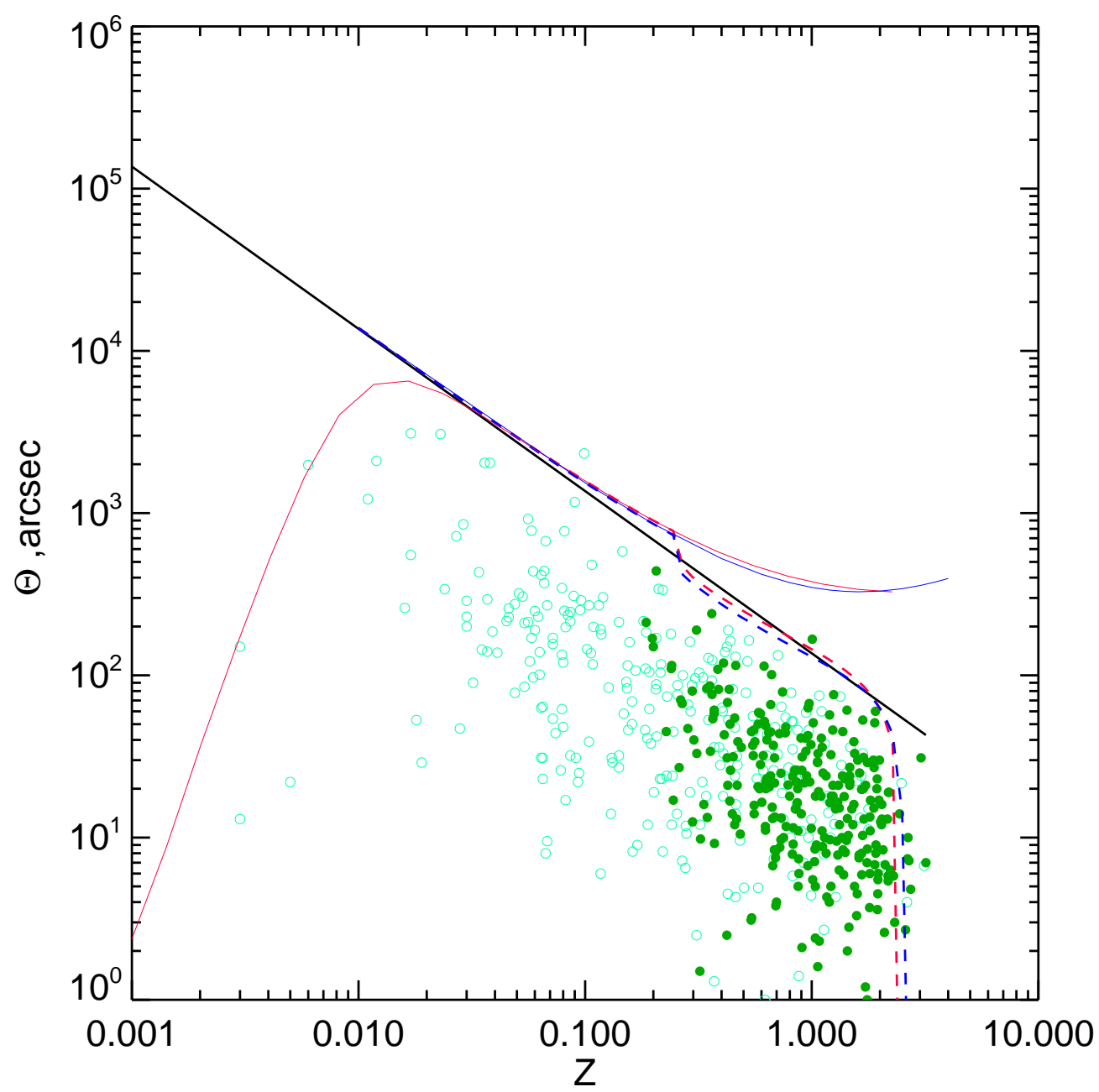

Figure 2. Angular size-redshift of Nilsson et al. [5]. Key: open cyan circles-radio galaxies, filled green circles QSOs. Black straight line - boundary of the Euclid static model. Red curve -standard boundary of the Friedman model, $\Omega=0.1$. Red dashed curve - boundary of the Friedman model with selection. Blue curve - standard boundary of the $\Lambda C D M$ model $\left(\Omega_{m}=0.27, \Omega_{v}=0.73\right)$. Blue dashed curve - boundary of the $\Lambda C D M$ model with selection.

constant $H_{0}=71 \mathrm{~km} / \mathrm{s} / \mathrm{Mpc}$; the largest radio source size $D_{\max }=2.8 \mathrm{Mpc}$; the highest radio source luminosity $L_{\max }=2 \times 10^{28} \mathrm{~W} / \mathrm{Hz}$ (this value corresponds to the highest radio source luminosity of the present catalog); deceleration parameter in the Friedman model $\mathrm{q}=0.05(\Omega=0.1)$. In the present $\Lambda C D M$ model $\Omega_{m}=0.27, \Omega_{v}=0.73$.

In addition to the radio sources, Fig. 2 shows:

1. relation $\Theta-\mathrm{z}$ in the Euclid static model (black straight line)

2. $\Theta$ - z relation in the Friedman model without the selection (red curve)

3. $\Theta-\mathrm{z}$ relation in the Friedman model with the selection (red dashed curve)

4. $\Theta$ - z relation in the $\Lambda C D M$ model without the selection (blue curve)

5. $\Theta$ - z relation in the $\Lambda C D M$ model with the selection (blue dashed curve).

The calculated selection adequately agrees with the upper limit of the radio source angular sizes in a wide range of radiation pattern parameters $(3): a=0.002 \div 0.01, \mathrm{n}=10 \div 20$. At that, the width of the radiation pattern at the 0.5 level ranges within $30 \div 21$. The best result can be achieved at $a=0.005$ and $\mathrm{n}=15$ (Fig. 2 ). The detection threshold $S_{t}=2.0$ Jy corresponds to the depth of the $4 \mathrm{C}$ survey [20,21]. It is clearly seen that the radio sources, as shown in [5], really lie below the "Euclid boundary"

$$
\Theta_{E}(z)=\frac{D_{\max }}{\frac{c z}{H_{0}}}
$$




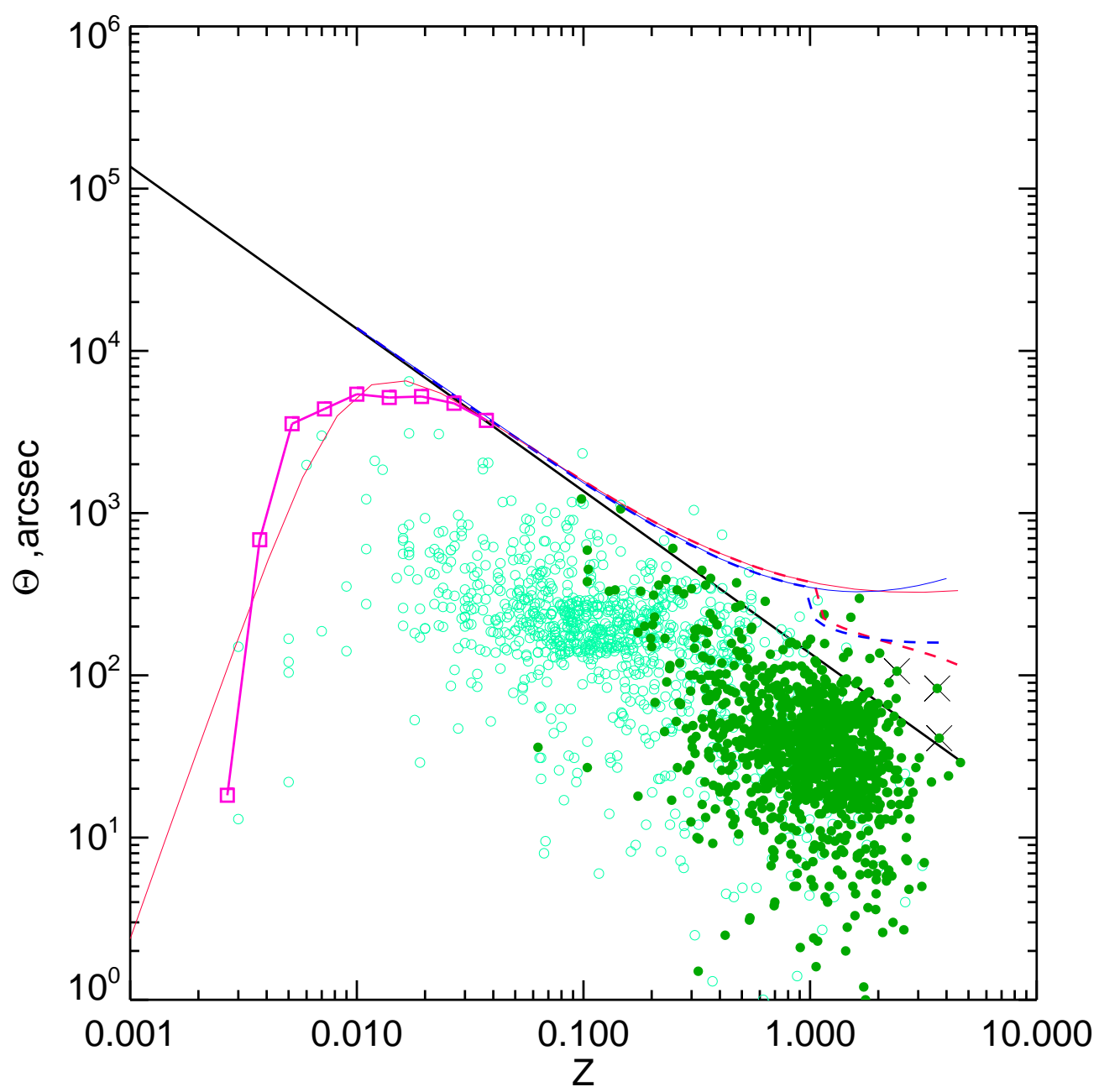

Figure 3. Angular size-redshift of the full catalog. Magenta squares+line-calculated boundary at $\mathrm{z}<0.1$. Other subscripts are as in Fig. 2.

The selection, which starts at $\mathrm{z}>0.25$, pressed the boundaries calculated by the standard formulas (lines 2 and 4) to the "Euclidean boundary" which agrees with the observations. Figure 3 shows the $\Theta-z$ diagram of the fully compiled catalog (subscripts identical to Fig. 2). Unlike Fig. 2, this diagram shows that the angular sizes of 43 radio sources overcame the "Euclid boundary" and are limited by the function $\Theta-z$ calculated in terms of selection (lines 3 and 5). The detection threshold here is determined by the deepest surveys.FIRST and NVSS. The minimum flux densities of the radio sources based on these surveys are $0.007 \div 0.015 \mathrm{Jy}$ which is in accordance with the threshold $S_{t} \approx 0.05 \mathrm{Jy}$ at $\nu=178 \mathrm{Mhz}$. The other parameters are the same as for Fig. 2. Hence, with the detection threshold decreased from $2 \mathrm{Jy}$ to $0.05 \mathrm{Jy}$, the selection shifted from $\mathrm{z}=0.25$ to $\mathrm{z}=0.95$ (Fig. 1). This allowed the observer to discover weak extended radio sources, the angular sizes of which at $z>0.05$ agree sufficiently with the $\Theta-z$ dependence in the standard space models. Naturally, such a result casts doubts on the obtained scale of the cosmological evolution of the radio sources size. The list of the radio sources which exceeded $\Theta_{E}(z)$ is given in the table 1. The doubtable objects have question marks. These three objects sufficiently agree with our concept of classical "double" radio sources. At the same time the author cannot rule out that they can be formed by the odd projection of the physically unrelated optical and radio components. In the redshift region less than 0.05 , where the radio galaxies are basically situated, both in Fig. 2 and in Fig. 3 the radio source maximal angular sizes are orders of magnitude smaller than the calculated ones. This is easily explained by the decrease of the space volume, and, consequently, by the decrease of the probability of the detection of an extended galaxy. This 
can be seen from the equation:

$$
N=\rho \Delta V(z) \int_{D_{z}}^{D_{\max }} p(D) d D
$$

Here $p(D) d D$ is the probability density function of the radio galaxy linear sizes, $\mathrm{N}$ is the number of galaxies larger than $D_{z}$ in the volume $\Delta V(z)$ at their space density $\rho$.

It is important that despite the 40 times reduction of the catalog minimum flux densities and the growth of the number of radio galaxies, the angular size boundary in this redshift region did not change. Hence, radio astronomers have catalogued the majority of close and most extended radio galaxies, and the angular size limitations at $\mathrm{z}<0.05$ are close to realistic. Let us use this boundary for the evaluation of

the space density of the radio galaxies. The upper limit of their linear sizes at some $\mathrm{z}$ and consequently their maximum angular size as a $\mathrm{z}$ function, are defined by solving the equation (5) for $D_{z}$. Let us assume the upper limit criterion as the condition that no more than one object can exceed the $D_{z}$ level, and define $\mathrm{N}=1$. In such a case it is not necessary to assume a more strict condition, defining $\mathrm{N}<1$. To solve the equation, we need to know the probability density distribution function of the radio galaxy linear sizes in the $\mathrm{z}<0.1$ region. We will define it in two ways: simple and not so simple. Let us start with the following assumptions: the space density of the radio galaxies and their linear size distribution function do not depend on the redshift. There are 392 radio galaxies with $\mathrm{z}<0.1$. The linear size distribution function of this sample, calculated with the help of the standard procedures, is shown in Fig. 4 (black curve). This very function we put under the integral in the equation (5). Further we assign the space density, divide the $\mathrm{z}$ range into several intervals, and, operating with the lower limit of the integral, solve the equation (5) in each interval. As a result, we obtain the dependence of $D_{z}$ on z. We change the space density $\rho$, then repeat the calculations and achieve the best visual fit of this curve with the radio galaxies apparent boundary in Fig. 3 (squares connected with the bold line). The best result is achieved for $\rho=10^{-4} M p c^{-3}$. The second way is simple. If we put the distribution function in the form

$$
p(D) d D \sim D^{\gamma} d D
$$

, then the equation (5) can be easily solved relative to $D_{z}$. We get a simple but cumbersome formula with which the $\Theta-z$ boundary was calculated, Figs. 2 and 3 (red curve). In this case, in order to adjust the calculations to the experiment we had to manipulate the parameters $\Theta-z$ and $\gamma$. In the author's subjective opinion the best result was achieved for $\rho=10^{-4} M p c^{-3}$ and $\gamma=-1.6$. If we adjust this boundary to the first variant, we need to define the parameters $\rho=8 \cdot 10^{-5} \mathrm{Mpc}^{-3}$ and $\gamma=-1.1$. The derived density estimate relates to the radio galaxies the luminosity of which is within the $3 \cdot 10^{21} \div 1.5 \cdot 10^{25} \mathrm{~W} / \mathrm{Hz}$ range. Let us focus on Fig. 4 again. The shown experimental probability density distribution function of the radio galaxy linear sizes is the distribution function of the visible sizes D. It is the result of the convolution of two functions: the real sizes distribution function $D_{o}$ and the distribution function of the radio galaxies orientation angles $\phi$ relative to the observer. The distribution function of these angles at a random space orientation of the radio sources is $\sin \phi d \phi$. Let the real sizes of the objects be between $D_{\min }$ and $D_{\max }$. Then, taking into account that $\sin \phi=\frac{D}{D_{o}}$, we obtain

$$
p(D) d D=D d D \int_{D}^{D_{\max }} \frac{P\left(D_{o}\right) d D_{o}}{D_{o} \sqrt{\left(D_{o}^{2}-D^{2}\right)}} .
$$

If $D<D_{\min }$ then $p(D) d D \approx D d D$, and depends on the distribution of the real sizes only weakly. Hence, the maximum of the distribution of the visible sizes is near $D_{\min }$. As the distribution function of the visible sizes in Fig. 4 has its maximum in the region of $0.2 \div 0.4 \mathrm{Mpc}$, one would expect that the minimum true sizes of the radio sources from this sample are in the same range. The catalog has been generated of 10000 objects, whose sizes are randomly distributed according to the $D_{o}^{-2.5} d D_{o}$ law within $0.25 \div 3.5 \mathrm{Mpc}$; their orientation angles comply with the distribution $\sin \phi d \phi$

Let us multiply the size of each object by its orientation angle sine, and we get the catalog of their apparent sizes. The distribution function of these sizes is shown in Fig. 4 (blue line). Its form in the range of $\mathrm{D}>$ Dmin is close to the real sizes distribution function. A reasonably good agreement between the distributions of the real and generated catalogs allows us to conclude cautiously that the minimum true size of the radio galaxies from the used list is close to $0.25 \mathrm{Mpc}$. 


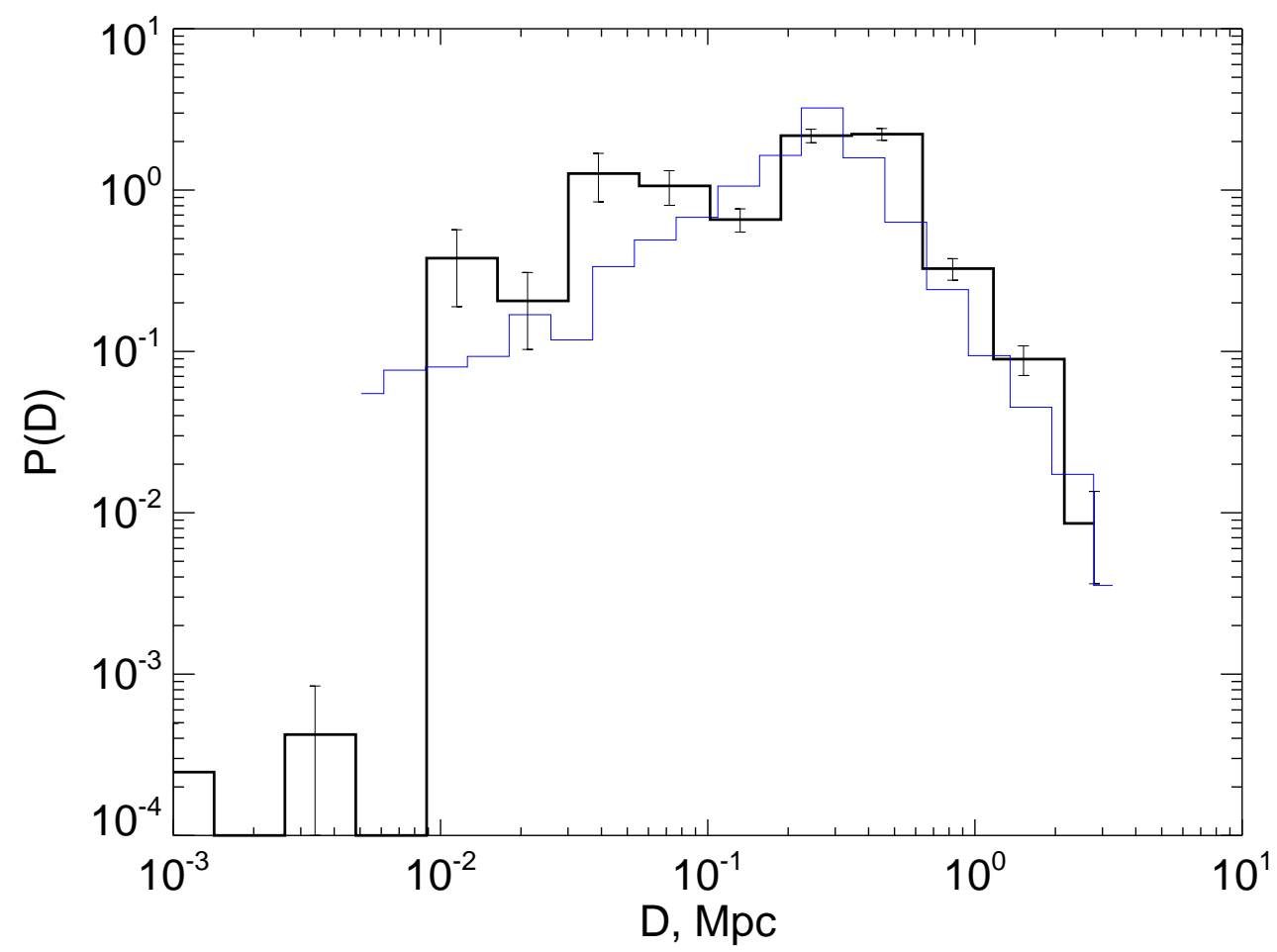

Figure 4. Distribution function of the visible sizes of the radio galaxies (black line) and of the generated objects (blue line).

table 1

\begin{tabular}{|c|c|c|c|c|}
\hline$\alpha_{2000}$ & $\delta_{2000}$ & $\Theta^{\prime \prime}$ & $z$ & $D(M p c)$ \\
\hline \multicolumn{5}{|c|}{ ilsson at al. [5] } \\
\hline $\begin{array}{lll}10 & 06 & 01.74\end{array}$ & $+34 \quad 54 \quad 10.4$ & 2332 & 0.0990 & 4.21 \\
\hline $14 \quad 32 \quad 15.54$ & $+15 \quad 48 \quad 22.4$ & 167 & 1.0050 & 1.34 \\
\hline \multicolumn{5}{|c|}{ Lara at al. $[12,13]$} \\
\hline $\begin{array}{lll}07 & 50 & 34.80\end{array}$ & +654126.6 & 192 & 0.7470 & 1.41 \\
\hline 082601.00 & +692037.0 & 432 & 0.5380 & 2.73 \\
\hline 195140.80 & +703740.0 & 312 & 0.5500 & 1.99 \\
\hline $2145 \quad 30.90$ & +81 $54 \quad 53.7$ & 1122 & 0.1460 & 2.84 \\
\hline \multicolumn{5}{|c|}{ IRST Amirkhanyan [15] } \\
\hline $\begin{array}{lll}08 & 38 & 13.32\end{array}$ & $+13 \quad 58 \quad 07.3$ & 137 & 2.0280 & 1.16 \\
\hline $08 \quad 56 \quad 25.56$ & +102033.8 & 43 & 3.7150 & $0.30 ?$ \\
\hline $1000 \quad 13.03$ & +102152.4 & 83 & 3.6390 & 0.61 \\
\hline $10 \quad 20 \quad 03.53$ & $\begin{array}{lll}-02 & 47 & 22.9\end{array}$ & 95 & 1.4470 & 0.81 \\
\hline $\begin{array}{lll}11 & 07 & 09.83\end{array}$ & +054743.6 & 78 & 1.8000 & 0.67 \\
\hline $\begin{array}{lll}11 & 19 & 27.99\end{array}$ & $+1302 \quad 49.3$ & 72 & 2.3980 & 0.59 \\
\hline $1214 \quad 31.40$ & $+18 \quad 28 \quad 13.8$ & 89 & 1.5900 & 0.76 \\
\hline $13 \quad 23 \quad 31.02$ & +545950.3 & 66 & 2.2080 & 0.55 \\
\hline $13 \quad 33 \quad 06.10$ & $+04 \quad 5105.1$ & 129 & 1.4050 & 1.10 \\
\hline $13 \quad 55 \quad 59.86$ & +19 $04 \quad 14.4$ & 90 & 2.2340 & 0.75 \\
\hline
\end{tabular}




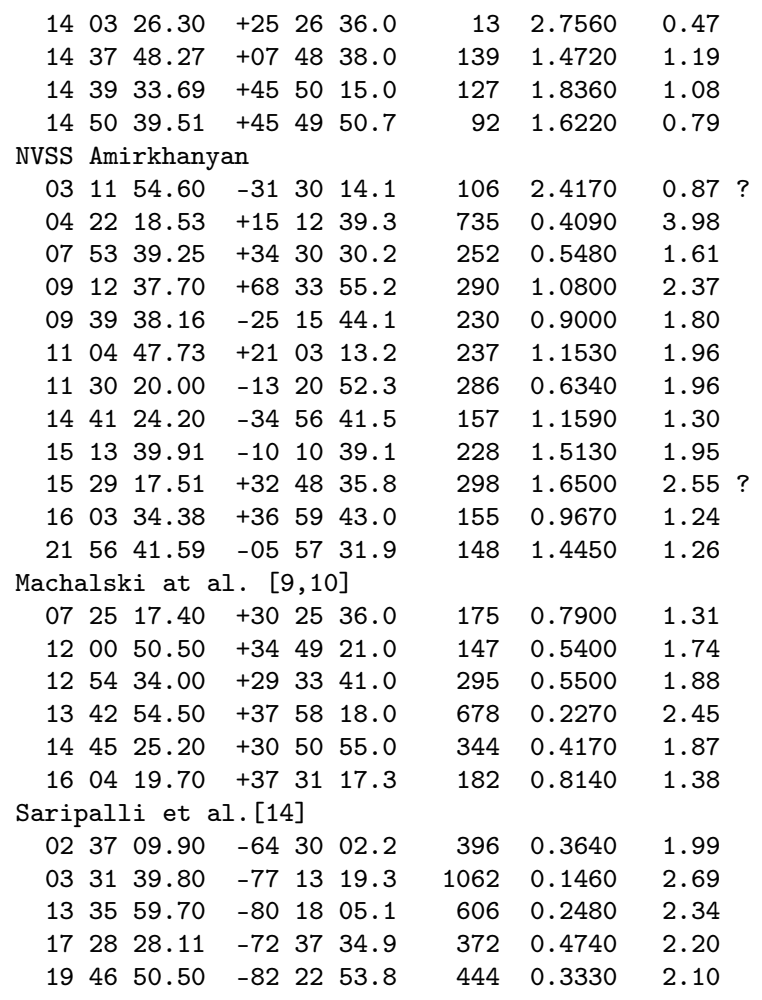

\section{CONCLUSIONS}

New observed data proves that the anisotropy of the radiation and structure of the radio sources cause a selection effect, placing an upper limit on the apparent angular sizes. If a depth of a survey is insufficient, the selection presses the boundary of the angular size - redshift test to the "Euclidean boundary". As a result, we see a contradiction between the boundaries in the standard models and in the observed data. Decreasing the catalog minimum flux density from 2.0 Jy to about $0.05 \mathrm{Jy}$ allowed us to shift the boundary of the selection by a factor of $4 \div 5$ in the direction of increasing $\mathrm{z}$. As a result, without the assumption of the cosmological evolution of the extended radio source physical sizes, an agreement was obtained between the observed $\Theta-z$ test boundary and the calculated one both in the Friedman model with $\mathrm{q}=0.05(\Omega=0.1$ ) and in the $\Lambda C D M$ model with $\Omega_{m}=0.27, \Omega_{v}=0.73$.

The analysis of the angular size boundary at $\mathrm{z}<0.1$ allowed us to evaluate the space density of close radio galaxies $\rho \approx 10^{-4} M p c^{-3}$.

\section{ACKNOWLEDGMENTS}

The author would like to thank D. V.Amirkhanyan for the substantial help in the work with the radio source images.

\section{REFERENCES}

1. F.Hoyle, IAU Symp. № 9, 529 (1959).

2. T.H.Legg, Nature 226,65 (1970).

3. G.K.Miley, Mon.Not.Roy.Astronom.Soc.152,477 (1971).

4. A.Hooley, M.S.Longair and J.M.Riley, Monthly Notices Roy. Astronom. Soc.182,127 (1978).

5. K.Nilsson et.all, Ap.J. 413, 453 (1993).

6. M. J. L.Orr and I. W. A. Brown, Mon.Not.Roy.Astronom.Soc.200,1067(1982). 
7. V. R. Amirkhanyan, Soobscheniya SAO 61,112 (1989).

8. V. R. Amirkhanyan, Astron. Zh. 70, 16 (1993).

9. J.Machalski, M.Jamrozy and S.Zola,Astron.Astrophys.371,445(2001).

10. J.Mashalski et al. , Ap.J.679,149 (2008).

11. A.Buchalter et al, Ap.J.494, 503 (1998).

12. L.Lara et.all, Astron.Astrophys.370, 409 (2001).

13. L.Lara et.all, Astron.Astrophys.378, 826 (2001).

14. L.Saripalli et al., Astron.J.130, 896 (2005).

15. V.R.Amirkhanyan, Astrophys. Bull. 64 ,325 (2009).

16. J.J.Condon et.all, Astron.J.115,1693 (1998).

17. R.L.White, R.H.Becker, D.J.Helfand and M.D.Gregg, ApJ 475,479(1997).

18. C.Fanti et al.,Astron. Astrophys, 528, 32,(2011).

19. I. S. Gradshtein and I. M. Ryzhyk, Tables of intergrals, summs, series, and products (Nauka,Moscow, 1971) [in Russian]. 20. J. D. Pilkington and J. F. Skott, Monthly Notices Royal Astron. Soc. 69, 183 (1965). 21. J. F. R. Gower, J. F. Skott, and D. Wills, Monthly Notices Royal Astron. Soc. 71, 49 (1967).

Translated by N. Oborina 